\section{That shrinking feeling}

Lab on a Chip: Miniaturisation for Chemistry and Biology

chair of editorial board Andreas Manz Royal Society of Chemistry. 4/yr. £395, \$596 (institutional); $£ 90, \$ 136$ (members of the Royal Society of Chemistry)

\section{Harold G. Craighead}

In recent years, miniaturization technologies, similar to those that produced the 'chips' of electronic integrated circuits, have been adapted to create biological sensors, devices for chemical analysis, and chemicalprocessing systems in a 'chip-like' format. A revolution similar to that in microelectronics may yield a proliferation of miniaturized chemical sensors, medical diagnostic devices and scientific research tools for the chemistry and biology communities. Increasing interest and activity in miniaturization technologies by industry, government labs and academic research groups has stimulated the need for, and the initiation of, the journal Lab on a Chip.

The aim of this journal is to provide a central resource for information and research results on miniaturization technology associated with chemistry and biology. The initial issues of the journal published a mixture of review articles and original research papers on the components and science associated with miniaturized chemical systems. It addressed 'plumbing', valves, materials and the control of fluid flow in scaled-down geometries or microfluidics. There were also papers on the methods of fabricating these microfluidic systems, which is an area of continuing development.

Lab on a Chip has attracted original papers and review articles from leading research groups. The articles are timely and of good technical quality. To date, the materials, fabrication methods and chemical applications appear to dominate the journal. Researchers and those who wish to stay informed of technological developments in these rapidly evolving areas may find it to be a significant resource. The articles are technical in nature and are appropriate for those with some technological background, as well as those actively working in the field. The journal should be attractive for researchers in the areas of microfluidics, sensors and microchemical analytical systems.

The editors of other technical journals in the areas of lab automation and chemical engineering are recognizing the importance of the miniaturization of analytical systems. Traditional scientific journals will continue to attract leading papers on the scientific advances in miniaturized systems, but $L a b$ on a Chip concentrates on the technological

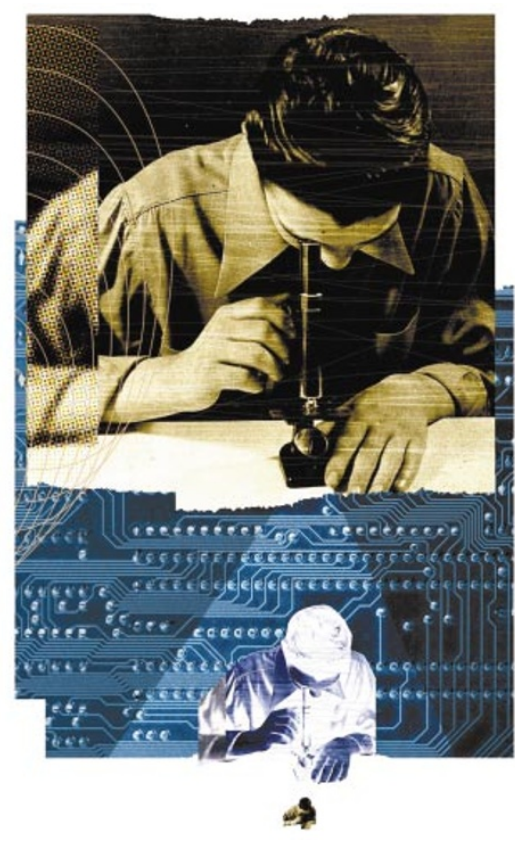

issues associated with fluid handling and the miniaturization of chemical systems. Its 'research highlights' section, which reviews recent publications in the area from other journals such as Nature, Analytical Chemistry, Sensors and Actuators and the Journal of Microelectrochemical Systems, is a helpful central source for keeping abreast of such developments.

Timely publication is important for the use of information in areas of rapid technological development. The journal has an electronic component and promises that papers will be available on the web 4-6 weeks before print publication. It accepts submissions electronically and aims for a rapid turnaround with publication in 120 days. Harold Craighead is in the School of Applied and Engineering Physics, Cornell University, Ithaca, New York 14853, USA.

www.rsc.org/is/journals/current/loc/ locpub.htm

\section{Biological molecules at large}

\section{Macromolecular Bioscience}

editor Ingrid Meisel

Wiley-VCH. 12/yr. 3,564 euros, $\$ 4,444$ (as

part of a journal package); 998 euros, $\$ 1,198$ (separate from 2003)

\section{David L. Kaplan}

There is increasing interest and research activity at the intersection of biological synthesis, polymer science, and materials science and engineering. This new journal, Macromolecular Bioscience, focuses on the interface between polymer science and the biological sciences. It aims to include studies from physical and chemical perspectives with relevance to biotechnology, as well as topics related to medical impacts. Physicists, chemists and engineers are the primary target audience.

The journal has been published within the Journal of Macromolecular Chemistry and Physics as a second section at the back, but from 2003 it will appear as a separate journal. This 'piggy-backing' is a useful approach to ensure wider visibility and to promote cross-fertilization from biological perspectives between the disciplines of chemistry and physics.

The summary page for each issue includes highlights such as titles, authors, abstracts and a key figure or table from each article, which facilitates the rapid scanning of contents. The contributions to the journal in the first year and a half emphasize biopolymer synthesis, enzymatic methods in polymer formation, phase control in biopolymers or hybrid synthetic/biosynthetic polymers, and related areas. Forays into topics such as tissue engineering, diagnostics and bioinformatics have not yet been featured, although they are proposed for broadening the scope of the journal.

The initial quality of contributions has been strong, and the inclusion of reviews, full papers and communications (short papers) is a selling point. The new journal was launched in February 2001 and nine issues have been published so far, with four to ten articles per issue. It will be published monthly from 2003, and the number of papers per issue probably needs to increase if it is to make an impact.

The journal has attracted a strong international advisory board that is actively contributing reviews and new papers to the journal, and this will clearly help to ensure short-term interest. Highlights include reviews on polyhydroxyalkanoates and block co-polymers from poly(L-lactide) and polyoxyethylene leading to nanostructures, and full papers have included studies of new cellulose derivatives, control of cholesterics from biopolymers, enzymatic digestion of new co-polymers of L-lactide and cyclic carbonates, synthesis of hydroxypropylcellulose, and enzymatic degradation of poly(L-lactide).

With the recent proliferation of journals focused on the theme of biological polymers, the likely long-term impact of this entrant into the field is not clear. However, the journal is off to a solid start and fits well within the Macromolecular Science series from Wiley-VCH. It might be useful to maintain the focus as currently offered in the contributions instead of broadening into the full range of proposed topics, because this will match most closely with the macromolecular chemistry and physics audience. 


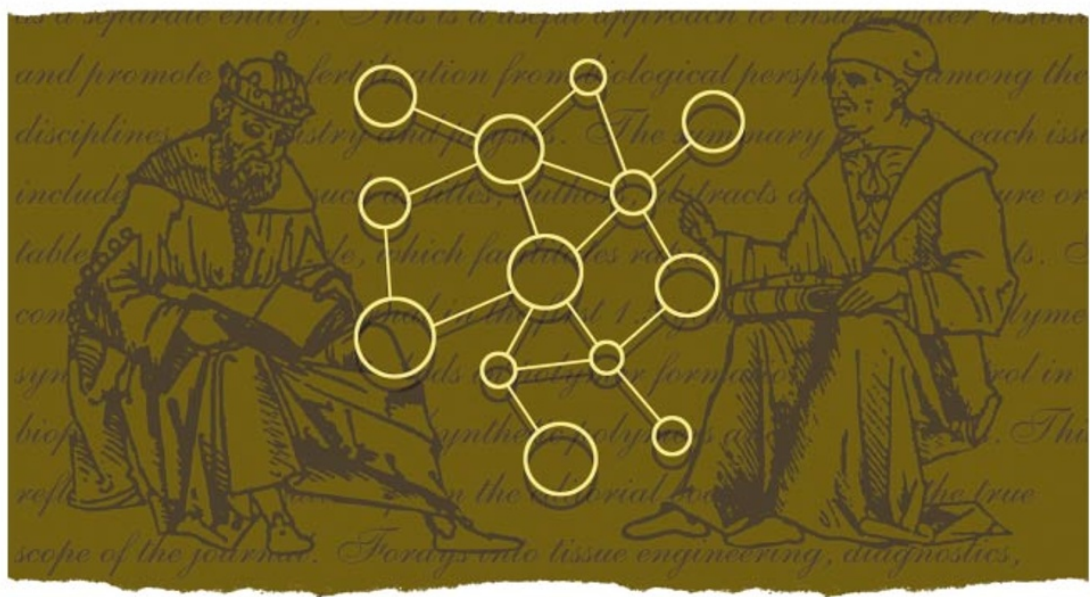

David L. Kaplan is in the Departments of Biomedical Engineering and Chemical o Biological Engineering, Tufts University, 4 Colby Street, Medford, Massachusetts 02155, USA. www.wiley-vch.de/publish/en/journals/ newJournals/2127

\section{A home for proteomics data?}

Molecular and Cellular Proteomics editor Ralph A. Bradshaw

American Society for Biochemistry and Molecular Biology. 12/yr. \$350, \$75

(members of the American Society for

Biochemistry and Molecular Biology), online access free this year

\section{Matthias Mann}

Suppose you have just finished an exciting study involving the identification of hundreds of proteins that are part of a complex, or that change in response to some condition. Where can you publish your results? This is a real problem. If you send them to a biological journal, the reviewers may tell you that that you should get functional data for the proteins, which could take you the rest of your career. If you send them to a technologically or analytically orientated journal, they will say that your methods have already been adequately described in the literature and so your paper is not very interesting. So you may just sit on your proteins, and have them worked up by incoming graduate students over the years, which would be a loss to the scientific community.

Enter Molecular and Cellular Proteomics $(M C P)$, a spin-off from the Journal of Biological Chemistry $(J B C)$, the official outlet for the American Society for Biochemistry and Molecular Biology. This new journal is dedicated to studies just like the one described above, and therefore meets a real need.

Judging from the articles published in the first few issues, the mission statement and the composition of the editorial board, $M C P$ takes an expansive view of proteomics, embracing subjects such as bioinformatics related to proteomics, protein databases, two-hybrid methods for protein interactions, two-dimensional gel studies, and mass-spectrometric methods.

There are three categories for original articles: research, database and technology. Another new feature is the online site, which, as well as displaying an electronic version of the printed journal, is intended to function as an associated database for the proteomics investigations described in the papers. This material can be much more extensive than usual, and $M C P$ is working on navigation and visualization features. The quality of papers published so far is good, with a few groundbreaking papers already in the bag.

Publication speed is, in my experience, quite rapid, with the additional advantage that accepted papers are put on the web immediately, even while the paper is being edited into the final version. This can be quite useful for authors in a publishing race.

Where should MCP stake its claim in the publishing food chain? The premier position in proteomics is taken by Nature Biotechnol$\circ g y$, and breakthrough biological results will also probably be reported elsewhere. MCP is well positioned to become to proteomics research what $J B C$ is to biological research -

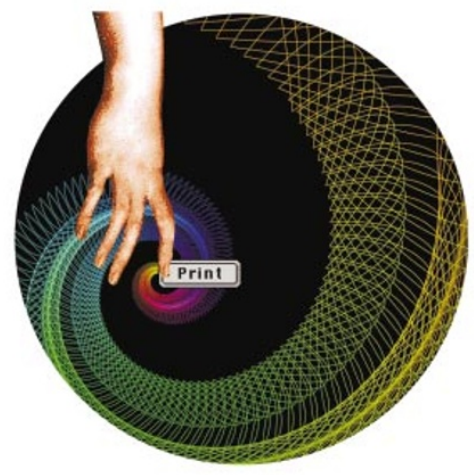

a standard place for solid results that have undergone stringent peer review and that will be easily accessible to almost everyone. For this to happen $M C P$ needs to maintain or even strengthen its reviewing standards, and focus on the quality of papers rather than the number published if it is to achieve an impact factor similar to that of $J B C$.

Once the journal is firmly established and its identity is clear in everybody's mind, there should be no shortage of papers as proteomics methods and proteomic-scale experiments become more commonplace. The Journal of Proteome Research, launched at almost the same time by the American Chemical Society, is likely to concentrate more on technological advances, and Electrophoresis and Proteomics will probably continue to be more focused on the twodimensional-gel community.

The format of MCP articles varies somewhat, and standardization would make the journal more visually appealing. In extension of its online features mentioned above, $M C P$ could perform a great service by helping to establish some standard as to how proteomics data collections are published and visualized so that they can actually be used by biologists.

In conclusion, faced with the dilemma I outlined at the start, I would encourage you to publish your proteins in MCP. Scientists with similar data should think 'out of the box' and submit their proteomics data for the community to use, and now there is a place for them to do it.

Matthias Mann is at the Protein Interaction Laboratory, University of Southern Denmark, Odense DK-5230, Denmark.

www.mcponline.org

\section{Shining a light on molecules}

\section{Photochemistry and \\ Photobiological Sciences}

editors-in-chief Frank Wilkinson \&

Tamás Vidóczy

Royal Society of Chemistry. 12/yr. £723, $\$ 1,091$

\section{Robin M. Hochstrasser}

The introduction of a new journal often signals the emergence of a sub-field or scientific direction that needs to establish a voice of its own, a place where specialists can air their views. A new journal can provide a balance when trends narrow the impact of journals that were intended to have a broader context. Specialist journals can also be healthily unimpressed with hyperbole, and tell it like it is. Photochemistry and Photobiological Sciences aims to do just this in fields related to "any 